\title{
Emphysematous cystitis and necrotizing fasciitis
}

\author{
Peter Zhan Tao Wang, MD; Paul R. Martin, MD; Patrick P.W. Luke, MD, FRCSC
}

Schulich School of Medicine \& Dentistry, University of Western Ontario, Division of Urology, Department of Surgery, London, ON

Cite as: Can Urol Assoc J 2014;8(7-8):e498-9. http://dx.doi.org/10.5489/cuaj.1865

Published online July 18, 2014.

\section{Abstract}

Emphysematous cystitis is an uncommon and often severe infectious condition of the bladder that usually affects women and diabetics. We report a case of a 62-year-old male patient who presented with concomitant emphysematous cystitis and necrotizing fasciitis of the right leg. The patient was initially managed with emergent disarticulation of the right leg. Tissue cultures were positive for Ciprofloxacin-resistant Klebsiella pneumoniae. He was treated with Piperacillin-Tazobactam; however, due to the failure of conservative management of the condition, the patient underwent a cystoprostatectomy with ileal conduit. To our knowledge, this is the first report of concomitant emphysematous cystitis and necrotizing fasciitis requiring aggressive surgical intervention for both diseases.

\section{Introduction}

As defined by Bailey in 1961, emphysematous cystitis (EC) is a complicated gas-forming urinary tract infection ${ }^{1}$ and is an uncommon and potentially life-threatening condition that requires prompt diagnosis and management. It typically presents with pneumaturia, hematuria, storage symptoms and abdominal pain with severe sepsis. Patients are asymptomatic in $7 \%$ of cases, with a median age at presentation of 66 years. ${ }^{2}$ Females are twice as likely to develop the condition as males. ${ }^{3,4}$ Diabetes mellitus (DM) was found to be a risk factor in two-thirds of the cases. ${ }^{2}$ Other risk factors include recurrent urinary tract infections, bladder outlet obstruction and neurogenic bladder. ${ }^{5}$ The most prevalent organism isolated in urinary cultures was Escherichia coli, followed by Klebsiella pneumoniae and Enterobactor aerogenes. ${ }^{2}$

\section{Case Report}

We report a case of a 62-year-old male who presented to the emergency department of a peripheral hospital with right leg pain. He was hemodynamically unstable and febrile and had a temperature of $38.6^{\circ}$. The patient was transferred to the intensive care unit at our institution after requiring intubation for a decreased level of consciousness.

The patient had a history of Klinefelter's syndrome, benign prostatic hypertrophy, recurrent Escherichia coli urinary tract infections, insulin-dependent DM and peripheral vascular disease, and his white blood cell count was elevated, at $16.5 \times 10^{9}$ cells/L. Physical examination demonstrated subcutaneous emphysema of the right thigh. The patient's external genitalia were unremarkable. A non-contrast computed tomography (CT) scan showed extensive subcutaneous air involving the entire right lower limb and tracking up into the pelvis, as well as air within the wall of the bladder and the prostate (Fig. 1). The patient underwent emergent right hip disarticulation for necrotizing fasciitis.

\section{Discussion}

The patient received broad-spectrum antibiotics prior to transfer to our institution; as a result, urine cultures and blood cultures were negative for growth. Tissue cultures from his right lower limb were positive for Klebsiella pneumoniae that was resistant to Ciprofloxacin. An attempt at conservative management with bladder drainage and Piperacillin-Tazobactam was made.

Reassessment at 48 hours demonstrated the failure of conservative management and a lack of clinical improvement. A repeat CT scan with contrast demonstrated persistent intramural air with new retroperitoneal air tracking up the right iliac fossa (Fig. 2). Consent was obtained and the patient subsequently underwent a cystoprostatectomy with ileal conduit. Final pathology from the cystoprostatectomy specimen demonstrated transmural necrosis. The patient convalesced well and was transferred back to home hospital after 13 days in the intensive care unit.

EC is predominantly diagnosed radiologically. Conventional imaging using a single plain film of the abdomen typically shows a curvilinear radiolucency outlining the bladder wall. The bladder mucosal surface may also have a "cobblestone" or "beaded necklace" appear- 


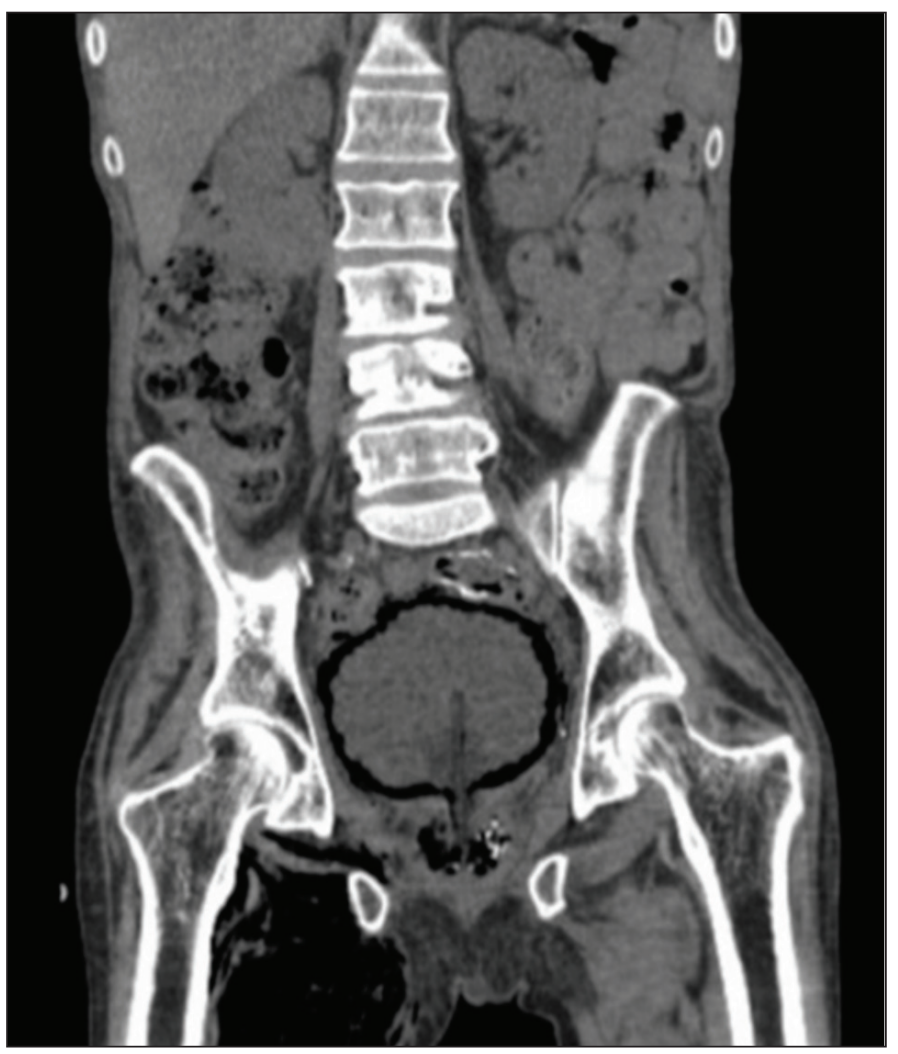

Fig. 1. A computed tomography scan of the abdomen and pelvis, without contrast. This coronal section shows subcutaneous air involving the entire right lower limb and intramural air in the bladder and prostate.

ance. ${ }^{5}$ Ultrasound shows diffuse bladder wall thickening with increased echogenicity. ${ }^{6}$ A CT scan is highly sensitive for detecting intraluminal and intramural gas, as well as for defining the extent and severity of EC. Intraluminal gas can occur with instrumentation, fistula necrosis or infection. However, intramural gas is most often associated with EC. ${ }^{2,5}$

\section{Conclusion}

The overall mortality rate is estimated at $7 \%$ in patients with $\mathrm{EC}$ alone; in patients with $\mathrm{EC}$ associated with another emphysematous infection, the mortality rate increases to $14 \% .^{2}$ Initial treatment involves adequate bladder drainage, broad-spectrum antibiotics and tight glycemic control. ${ }^{2}$ Ten percent of patients with EC cannot have their condition controlled by medical therapy and require surgical intervention. ${ }^{4}$ In the literature, there has been one other case report of a patient with concomitant necrotizing fasciitis and EC.

The patient underwent debridement of the left hip without requiring disarticulation or surgical intervention for his EC. ${ }^{6}$

Competing interests: Dr. Wang, Dr. Martin and Dr. Luke declare no competing financial or personal interests.

This paper has been peer-reviewed.

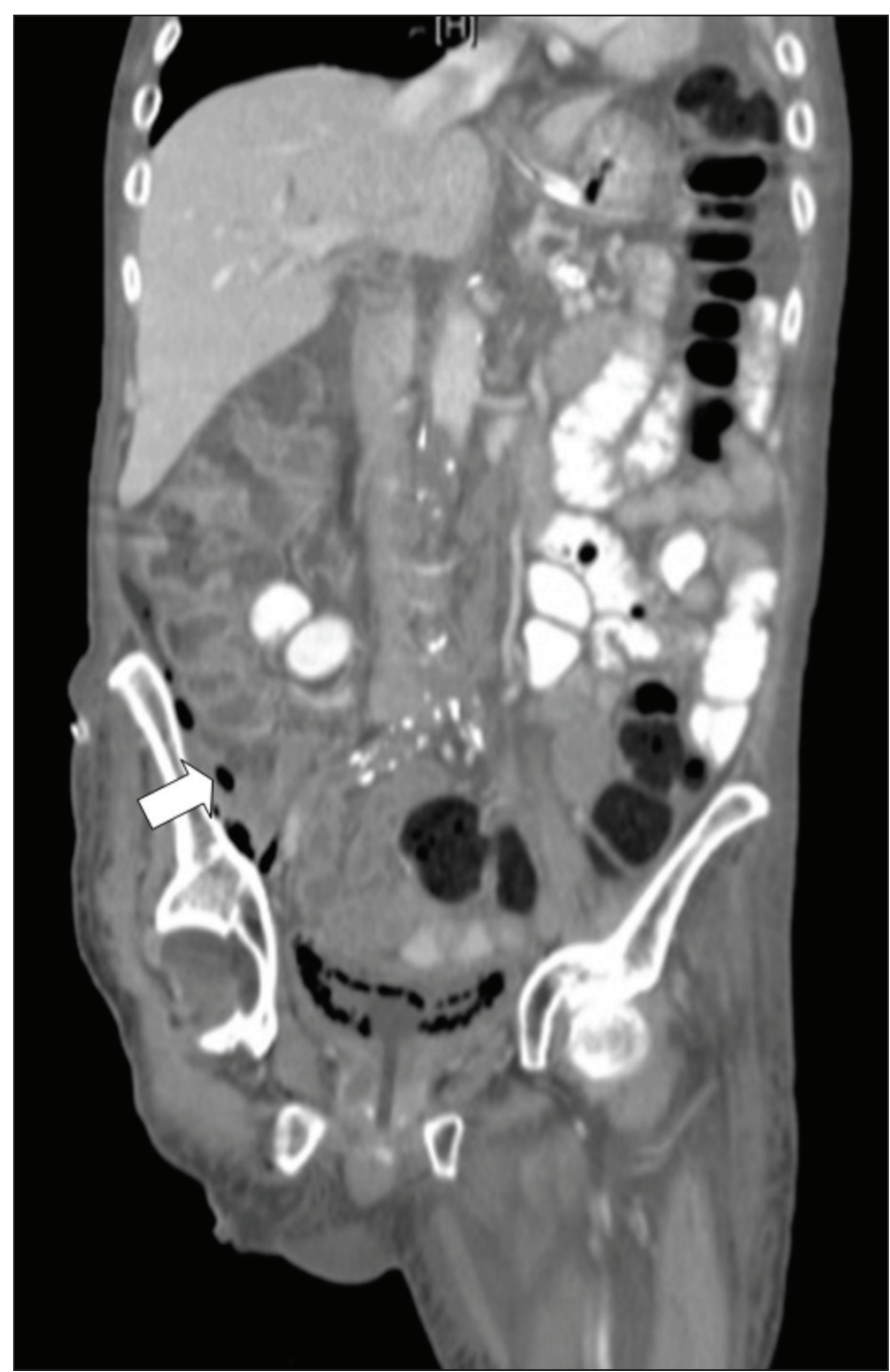

Fig. 2. A computed tomography scan of the abdomen and pelvis with oral and intravenous contrast post-right hip disarticulation. This coronal section shows new retroperitoneal air in the right iliac fossa and persistent intramural bladder air.

\section{References}

1. Bailey H. Cystitis emphysematosa: 19 cases with intraluminal and interstitial collections of gas. Am J Roentgenol Radium Ther Nucl Med 1961;86:850-62.

2. Thomas AA, Lane BR, Thomas AZ, Remer EM, Campbell SC, Shoskes DA. Emphysematous cystitis: A review of 135 cases. BJU Int 2007;100(1):17-20.

3. Patel NP, Lavengood RW, Fernandes M, Ward JN, Walzak MP. Gas-forming infections in genitourinary tract. Urology 1992;39:341-5.

4. Quint HJ, Drach GW, Rappaport WD, Hoffmann CJ. Emphysematous cystitis: A review of the spectrum of disease. J Urol 1992;147(1):134-7.

5. Grayson DE, Abbott RM, Levy AD, Sherman PM. Emphysematous infections of the abdomen andpelvis: A pictorial review. Radiographics 2002;22(3):543-61.

6. Chen CC, Su YC, Hou SW, Hung SW, Chong CF. Emphysematous cystitis combined with necrotizing fasciitis. Intern Med J 2006;36(8):536.

Correspondence: Dr. Patrick Luke, Professor, Department of Surgery, Division of Urology, Western University, University Hospital, Room C4-207, 339 Windermere Rd, London, ON, N6A 5A5; fax: 519-663-3858; patrick.luke@hsc.on.ca patrick.luke@hsc.on.ca 